Trauma Berufskrankh $2007 \cdot 9$ [Suppl 3]:

S349-S350

DOI 10.1007/s10039-007-1312-6

Online publiziert: 9. November 2007

(c) Springer Medizin Verlag 2007

H. Windhagen

Orthopädische Klinik der Medizinischen Hochschule Hannover im Annastift, Hannover

\title{
Der Hüftoberflächenersatz - mehr als eine Mode?
}

zogenes Knochengerüst zurück, das im Rahmen der Propriozeption eine wesentliche Rolle spielen kann. Forschungen zu diesem Thema sind bisher jedoch kaum publiziert. Oberflächenprothesen existieren in zementfreien und zementierten Versionen mit unterschiedlich langen Zentrierstiften. Die Passqualität von Prothesen kann erheblich variieren.

Metall-Metall-Paarung. Die Gleitpaarung von Hüftoberflächenprothesen besteht aus einer Metall-Metall-Paarung aus Kobaltchrom. Die Metallbelastung von Patienten mit Metall-Metall-Paarungen ist in der Vergangenheit hinreichend belegt. Es muss davon ausgegangen werden, dass die Blutserumwerte von Kobalt und Chrom sowie Nickel höher als bei einer nichtversorgten Bevölkerung sind. Interessant ist dabei ein anfängliches Einschleifen von Pfanne und Hüfte mit einer höheren Metallbelastung, die dann im Verlauf mehrerer Monate zurückgeht. Die Gleitpaarung Metall-Metall beim Hüftoberflächenersatz ist auch tribologisch interessant, da die Gelenkschmierung durch Großköpfe nicht immer positiv beeinflusst wird. Neuere Entwicklungen weisen Vertiefungen im Bereich der Kappenoberfläche (Golfballprinzip) auf, die die Funktion von Schmiertaschen übernehmen und dazu beitragen, den Gleitfilm innerhalb dieser Großpaarung dauerhaft zu erhalten.

Andere Materialpaarungen. KeramikKeramik-Paarungen sowie Keramik-PEPaarungen sind in der präklinischen und klinischen Testung und werden möglicherweise die nächste Generation der Hüftkopfkappen darstellen.

\section{Implantationstechnik}

Die Implantation von Hüftkappen verlangt eine exakte Positionierung der Kappe auf dem Femurkopf. Dazu werden Teile des Femurkopfs abgefräst und ein Zentrierstift eingebracht. Als Standardverfahren wird die Vermessung des Schenkelhalses angesehen, wobei mit Ausrichthilfen versucht wird, eine in allen Ebenen möglichst gute Zentrierung des Stifts im Schenkelhals zu erreichen. Vernachlässigt wird dabei die anatomisch nicht exakte Position des Kopfs auf dem Schenkelhals. Die anatomische Positionierung des Kopfs bedeutet eine asymmetrische Verlagerung nach medial und dorsal. Bei der von uns angewendeten Implantationstechnik wird die Geometrie des Schenkelhalses nur zur Impingementvermeidung herangezogen; die Bestimmung des Zentrums erfolgt durch einen extern von lateral kaudal nach medial kranial geführten Zentrierdraht unter dem Bildwandler und nachfolgende Aufbohrung. Diese elegante Technik (Wirth et al. [2]) ist für viele Chirurgen gut nachvollziehbar, da eine ähnliche Technik bei Anbohrung des Hüftkopfs sowie der Schenkelhalsfrakturversorgung angewendet wird. Nachdem eine zentrale Bohrung im Schenkelhals erfolgt ist, wird ein lateraler Zugang eröffnet, der mit einer großzügigen azetabulumnahen Kapselinzision verbunden ist. Danach lässt sich der Hüftkopf ohne Schädigung des Glutaeus medius luxieren und abfräsen. Der relativ große Kopf verhindert häufig den Weg zur Pfanne, was durch die Kapselinzision verbessert werden kann. Luxation sowie Positionierung des Kopfs und Schenkelhalses bedürfen während der Pfannenfräsung besonderer Aufmerksamkeit, da die kopfversorgenden Gefäße sehr häufig fensensibilität bleibt durch die Erhaltung des Hüftkopfs ein von Osteozyten durch- 
komprimiert werden. Nach Fräsung der Pfanne erfolgt der Größenabgleich von Pfanne und Kopf, danach die Entfräsung des Kopfs, das Aufsetzen der Kappe sowie eine ausführliche Resektion von Osteophyten und das Abgleichen der Taillierung von Schenkelhals und Kopf zur Impingementvermeidung.

\section{Ergebnisse}

Seit 2004 wurden bei unseren Patienten 108 zementfreie Kappen BS (ESKA, Lübeck) eingebracht. Der Vergleich der postoperativen Beinlänge und des Offsets von 4 Hüftendoprothesensystemen wurde prospektiv begleitet. Hier konnte im Vergleich eines Standardschafts (Bicontact, Aesculap, Tuttlingen), einer Schenkelhalsprothese (CUT, ESKA, Lübeck) und einer Kurzschaftprothese (METHA, Aesculap, Tuttlingen) festgestellt werden, dass das Offset nach Kurzschaftprothesenimplantation am geringsten ausgeprägt war und sich Standardschaft und Kopfkappe gleich verhielten. Im Bereich der Pfanne konnten keine wesentlichen Unterschiede bezüglich der Pfannenhöhe festgestellt werden. Sowohl für die Kurzschaftprothese als auch für die Kopfkappe wurde eine diskrete Beinverlängerung um $>10 \mathrm{~mm}$ festgestellt. Die exakteste Beinlängeneinstellung war mit dem Standardschaft möglich. Nach der Implantation einer Kopfkappe kam es in 2,4\% der Fälle zu Schenkelhalsfrakturen. Insbesondere der Ausschluss von Patienten mit schlechter Knochenqualität und die Verbesserung des Instrumentariums mit Sicherheitsfräsen führte im Verlauf der 4 Beobachtungsjahre zu einer deutlichen Reduktion des Kopfversagens mit nachfolgender Fraktur. Die häufigsten postoperativen Beschwerden wurden bei bis zu 6\% der Patienten als reduzierter Bewegungsumfang ohne und mit Schmerzen beschrieben. Einschränkungen der Beweglichkeit mit Schmerzen wurden als Impingementproblematik definiert und näher analysiert.

\section{Diskussion}

Insbesondere für die Impingementvermeidung erwies es sich als bedeutsam, einen großen Abstand zwischen der anterolateralen Schenkelhalsposition und der anterolateralen Kappenkante zu finden. Diese Differenz definiert die Basis für das anterolaterale Anschlagen und nachfolgende Impingement. Entsprechend des Konzepts des $\boldsymbol{\alpha}$-Winkels nach Nötzli et al. [3] muss die Kurvatur der Kappe bis zu einem Winkel von $55^{\circ}$ laufen, um ein anterolaterales Anschlagen zu verhindern. Ein Winkel über $55^{\circ}$ kann wie ein CAM-Impingement angesehen werden und führt zu Einschränkungen in der Beweglichkeit und möglichen Impingementschmerzen. Das Prinzip der Impingementvermeidung durch Osteophytenentfernung, kraniale Resektion oder größere, kopforientierte Kappen muss als Grundprinzip für eine erfolgreiche Kappenimplantation angesehen werden. Die Ergebnisse dieser Studie zeigen, dass das Prinzip der Kappe funktioniert und dass Komplikationen hauptsächlich chirurgisch bedingt sind, entweder in Form einer zu geringen Pfannenöffnung und -inklination oder einer nicht beseitigten Impingementproblematik am Schenkelhals.

Um das Versagen von Hüftkopfkappen zu verhindern, ist eine aufwendige Selektion der Patienten notwendig. Das beinhaltet eine präoperative magnetresonanztomographische Diagnostik, die eine Nekrose des Hüftkopfs ausschließt. Gleichzeitig empfiehlt sich eine Osteoporosediagnostik. Bei Abweichungen von mehr als einer Standardabweichung sollte keine Kappenimplantation durchgeführt werden. Eine zu geringe Schenkelhalskopfdifferenz anterolateral, die sich etwa als Erhöhung des $\alpha$-Winkels über $55^{\circ}$ ausdrückt, muss ebenfalls als Kontraindikation zur Kopfkappe angesehen werden. Beim Zugang ist der Gefäßversorgung des Hüftkopfs besondere Aufmerksamkeit zu schenken. Dazu gehört der laterale Zugang, der die dorsalen Arteriae circumflexae femoris intakt lässt und anders als der posteriore Zugang hier prinzipiell bessere Voraussetzungen bietet. Gleichzeitig muss von einer Kompression der Arteriae circumflexae femoris bei forcierter Außenrotation ausgegangen werden, so dass ein azetabulumnahes, dorsales Kapselrelease und eine entsprechende Lagerung ohne wesentliche $\mathrm{Au}$ ßenrotation zur Pfannenfräsung zu einer besseren Kopfdurchblutung führen.
Fazit

Der Oberflächenersatz der Hüfte ist ein relativ neues Verfahren und sollte nur bei Patienten angewendet werden, die auch nachhaltig von einem knochensparenden Endoprothesenkonzept profitieren, was eher für Patienten unter 60 Jahren gilt. Die gegenwärtigen klinischen Studien lassen eine kritische Indikation bei älteren Patienten noch nicht zu. Aufgrund noch unbekannter langfristiger Standzeiten sowie leicht höheren Komplikationsraten als bei der Standardendoprothetik bleibt der Standardschaft als endoprothetische Versorgung nach wie vor das Mittel der Wahl für Patienten im höheren Alter. Für junge und sehr junge Patienten bietet die Kappe jedoch die Möglichkeit, durch knochensparende Verfahren Zeit zu gewinnen und bei zukünftigen Endoprothesenwechseln bessere Alternativen zu haben. Eine umfassende Aufklärung des Patienten über noch unbekannte Standzeiten, Metallbelastungen, Komplikationsrisiken und insbesondere Erfolge der Standardendoprothetik sind unverzichtbar.

\section{Korrespondenzadresse}

\section{Prof. Dr. H. Windhagen}

Orthopädische Klinik im Annastift, Medizinische Hochschule Hannover, Anna-von-Borries-Straße 1-7, 30625 Hannover windhagen@annastift.de

Interessenkonflikt. Der korrespondierende Autor weist auf folgende Beziehung/en hin: Wissenschaftlicher Beirat der Firma ESKA

\section{Literatur}

1. Itayem R, Arndt A, McMinn DJ et al. (2007) A fiveyear radiostereometric follow-up of the Birmingham Hip Resurfacing arthroplasty. J Bone Joint Surg Br 89: 1140-1143

2. Wirth CJ, Gosse F (2006) Improved implantation technique for resurfacing arthroplasty of the hip. Oper Orthop Traumatol 18: 214-224

3. Nötzli HP, Wyss TF, Stoecklin CH et al. (2002) The contour of the femoral head-neck junction as a predictor for the risk of anterior impingement. J Bone Joint Surg Br 84: 556-560 